\title{
Avaliação clínico-laboratorial de uma preparação de insulina suína lenta no controle de cães diabéticos
}

\author{
Laboratorial and clinical evaluation of a lente porcine insulin preparation in diabetic dogs
}

\author{
Alan Gomes Pöppl ${ }^{1,3}$, Simone Tostes de Oliveira ${ }^{1,2}$, Marcus Streb Sortica', Rafael Rodrigues Ferreira', \\ Patrícia Rick Barbosa², Luciana de Almeida Lacerda² \& Félix Díaz González ${ }^{2}$
}

\begin{abstract}
RESUMO
A maioria dos cães diabéticos necessitam de insulinoterapia após realizado o diagnóstico da doença, independentemente dos eventos envolvidos na etiologia do problema, a fim de controlar os sinais clínicos, retomar a qualidade de vida e evitar a cetoacidose. A insulina NPH humana tem sido utilizada em duas aplicações diárias, mas terapias alternativas com uma aplicação diária têm sido propostas. O objetivo deste trabalho foi avaliar a resposta clínico-laboratorial de cinco cães a uma terapia insulínica alternativa, com uma preparação de insulina suína lenta SID. Refeições foram administradas no momento da aplicação de insulina e após oito horas, com jejum de dezesseis horas até a nova refeição e aplicação de insulina. Dois pacientes já recebiam insulina NPH humana BID antes do estudo. Outros três iniciaram com insulina lenta suína após o diagnóstico de diabetes. $\mathrm{O}$ acompanhamento dos pacientes foi realizado durante dois meses mediante hemograma, urinálise e painel bioquímico. Os melhores resultados foram alcançados em uma cadela que sofrera ovariohisterectomia e mastectomia, recebendo ração comercial rica em fibras. Em geral, observou-se um razoável controle dos sinais clínicos e laboratoriais da diabetes, que foi menos eficiente em decorrência de fatores como estresse, estro/diestro e doenças intercorrentes como hipotireoidismo. Seria recomendável o uso de insulina lenta suína para cães em casos em que tenham sido eliminadas possíveis causas de resistência à insulina (desordens hormonais, inflamatórias, infecciosas ou neoplásicas), associado a dieta rica em fibras para melhores resultados.
\end{abstract}

Descritores: diabetes mellitus canina, insulina lenta suína.

\section{ABSTRACT}

Almost all diabetic dogs need insulin therapy after a diagnosis of diabetes mellitus independently which etiology factors are involved, as a way for controlling clinical signs, recovering life quality and avoiding diabetic ketoacidosis. Human NPH insulin have been used twice daily for treatment of diabetic dogs, but other alternative therapy using administration once daily has been proposed. The aim of this work was to evaluate five diabetic dogs using clinical and laboratorial responses to an alternative porcine lente insulin therapy used once daily. Meals were offered at insulin application and after eight hours, with sixteen hours fasting before new insulin application and meal. Two patients already received human NPH insulin twice daily before entering in the new therapy. Other three dogs started with porcine lente insulin after the initial diagnosis of diabetes. Patients were monitored for two months using complete blood counts, urinalysis and biochemical panel. Best results were observed in a neutered, mastectomized bitch receiving high fiber diet. Patients that were already undergoing insulin therapy showed better control with human NPH insulin. In general, a reasonable control of clinical and laboratory traits of diabetes was observed, which was less efficient when other factors as stress, estrus/diestrus and concurrent diseases such hypothyroidism were present. This type of insulin could be recommended in dogs that have eliminated other possible causes of insulin resistance, like endocrine, inflammatory, infectious or neoplasic disorders, as well as associated to a high fiber diet to achieve better results.

Key words: canine diabetes mellitus, porcine lente insulin. 


\section{INTRODUÇÃO}

Independente dos fatores etiológicos envolvidos no desenvolvimento da diabetes mellitus canina [10], muitos dos quais são similares à etiologia da diabetes mellitus tipo II humana [4], no momento do diagnóstico os pacientes apresentam necessidade de insulinoterapia para controlar os sinais clínicos, retomar a qualidade de vida e evitar a cetoacidose diabética [5,9].

Existem diversas apresentações de insulina para tratamento de pacientes diabéticos com variações tanto na espécie de origem da insulina como na duração do efeito e na potência (na ordem de menor potência e maior duração do efeito a maior potência e menor duração do efeito: Glardine, ultralenta, zinco protamina, lenta, NPH, mistas de NPH e regular cristalina, regular cristalina, Lispro, Aspart) [4,18,31]. Para tratamento de cães diabéticos preconiza-se a utilização de insulina humana NPH ou lenta (a cada 12 horas) por sua fácil obtenção no mercado, reduzida antigenicidade e adequada farmacodinâmica, com início do efeito de 30 a 120 minutos após a aplicação e duração média do efeito de 12 horas [5,31].

A insulina humana difere em apenas um aminoácido da insulina canina e a formação de anticorpos parece incomum após longos períodos de administração [4]. Em contraste, a insulina bovina difere em dois aminoácidos, e é altamente antigênica em cães [3,6]. A insulina suína apresenta a mesma sequiência de aminoácidos da insulina canina, constituindo a forma ideal de tratamento em cães diabéticos pela ausência de anticorpos anti-insulina [4].

O objetivo deste trabalho foi avaliar a resposta clínico-laboratorial de cães diabéticos a uma terapia alternativa com uma preparação de insulina suína lenta utilizada uma vez por dia.

\section{MATERIAIS E MÉTODOS}

Cinco cadelas diabéticas participaram do estudo. Os proprietários autorizaram a participação de seus animais mediante termo de consentimento informado. Três pacientes iniciaram a terapia insulínica após o diagnóstico enquanto outras duas já estavam em tratamento com insulina humana NPH duas vezes por dia e passaram a receber insulina suína lenta uma vez por dia. A implementação da terapia com insulina suína lenta ${ }^{1}$ seguiu as instruções do fabricante a respeito da dosificação inicial (1 UI/kg mais dose suplementar conforme o peso de 1 UI para cães com menos de $8 \mathrm{~kg}, 2$ UI para cães entre 8 e 12 kg, 3 UI para cães entre 12 e $20 \mathrm{~kg}$ e 4 UI para cães com mais de 20 kg), bem como ao regime alimentar (uma refeição junto com administração de insulina e outra refeição cerca de 8 horas depois, seguindo-se um jejum de 16 horas até próxima refeição e aplicação de insulina).

Após consulta inicial para início do tratamento, os pacientes foram reavaliados semanal ou quinzenalmente, conforme o caso, durante dois meses, mediante revisão clínica, hemograma, urinálise e painel bioquímico. Amostras de sangue foram colhidas em tubos com EDTA $10 \%$ para hemograma e em tubos sem anticoagulante para análises bioquímicas. $\mathrm{O}$ soro obtido por centrifugação foi conservado a $-20^{\circ} \mathrm{C}$ até a realização das análises. As coletas de urina foram realizadas por micção natural ou cateterização, mantendo a urina refrigerada $\left(5^{\circ} \mathrm{C}\right)$ até sua análise, efetuada em no máximo duas horas após a coleta.

A quantidade total de eritrócitos, leucócitos e hemoglobina foi determinada por diluição do sangue e leitura em contador automático ${ }^{2}$. O hematócrito foi obtido por centrifugação do sangue total em tubo capilar (10.000 rpm por 5 minutos). Os valores de VGM e CHGM foram calculados [34]. A leucometria diferencial foi determinada por leitura de esfregaço sangüíneo corado pelo método Panótico rápido. $\mathrm{Na}$ análise da urina, foi determinada a densidade com refratômetro e o exame químico com uso de tiras reagentes ${ }^{3}$. O exame do sedimento urinário foi realizado após centrifugação de $10 \mathrm{ml}$ de urina (1.500 rpm por $10 \mathrm{minu}-$ tos) e observação ao microscópio óptico (400x). Todas as análises bioquímicas foram realizadas por espectrofotometria com kits diagnósticos e contemplaram avaliação da atividade sérica das enzimas ALT (alaninaaminotransferase), AST (aspartato-aminotransferase), GGT ( $\gamma$-glutamil-transferase), FA (fosfatase alcalina), CK (creatina quinase), amilase pancreática e mensuração sérica de albumina, proteínas totais, uréia, creatinina, fructosamina, colesterol, triglicerídeos, potássio e bilirrubina total. Globulinas foram calculadas a partir dos valores de proteínas totais menos a albumina. A mensuração da glicemia foi realizada ambulatorialmente antes da aplicação de insulina com glicômetro portátil $1^{5}$.

\section{RESULTADOS}

\section{Paciente 1}

Poodle, fêmea, 10 anos de idade e diagnóstico de diabetes mellitus cerca de 6 meses antes. Teve tratamento prévio com insulina NPH humana (2 UI a 
cada 12 horas), alimentada com ração comercial rica em fibras com adição de peito de frango desfiado. Havia sofrido ovariohisterectomia e mastectomia em decorrência de adenomas mamários. Apesar de encontrar-se em tratamento prévio, a paciente apresentava fraco controle glicêmico antes do início da terapia insulínica com Caninsulin (Tabela 1). Nas revisões após 10 e 28 dias evidenciou-se quadro de cistite tratada com Enrofloxacina $5 \mathrm{mg} / \mathrm{kg}$ BID, após realização de cultura urinária e antibiograma. A dose de Caninsulin foi aumentada para 8 UI após 44 dias de tratamento ao observar-se hiperglicemia persistente superior a 300 $\mathrm{mg} / \mathrm{dL}$ antes da aplicação de insulina pela manhã, embora tivesse melhorado os sinais clínicos. A paciente chegou ao final do estudo com bom controle glicêmico após 60 dias de acompanhamento. Os achados referentes aos hemogramas, urinálises e perfis bioquímicos obtidos ao longo do período de 117 dias de acompanhamento da paciente são apresentados na Tabela 1. A última avaliação foi realizada 57 dias após retomar terapia insulínica com insulina NPH humana 2 UI BID.

Tabela 1. Parâmetros clínico-laboratoriais na paciente 1 em diferentes períodos após tratamento com Caninsulin SID, e após 57 dias de retomar a terapia insulínica com insulina humana NPH BID.

\begin{tabular}{|c|c|c|c|c|c|c|c|}
\hline & Dia 0 & Dia 10 & Dia 28 & Dia 44 & Dia 60 & $\begin{array}{l}57 \text { dias } \\
\text { após NPH }\end{array}$ & $\begin{array}{l}\text { Valores de } \\
\text { referência }\end{array}$ \\
\hline Peso corporal (kg) & 6,3 & 6,2 & 5,8 & 6,2 & 6,0 & 6,0 & \\
\hline Sinais clínicos & PF/PD/PU & NF/PU/PD & NF/NU/ND & $\mathrm{PF} / \mathrm{NU} / \mathrm{ND}$ & NF/NU/ND & NF/NU/ND & NF/NU/ND \\
\hline Hemograma & s/a & s/a & s/a & s/a & $\mathrm{s} / \mathrm{a}$ & s/a & $\#$ \\
\hline \multicolumn{8}{|l|}{ Urinálise } \\
\hline Densidade & 1.058 & 1.048 & 1.045 & 1.045 & 1.045 & 1.045 & $1.020-1.045$ \# \\
\hline Glicosúria (mg/dL) & 500 & 1000 & 500 & 1000 & 1000 & 1000 & - \# \\
\hline Cetonúria (mg/dL) & - & 5 & 5 & 5 & 5 & 5 & - \# \\
\hline Sedimento & - & $\mathrm{Pi} / \mathrm{Ba}$ & $\mathrm{Pi} / \mathrm{Ba} / \mathrm{He} / \mathrm{Pr}$ & Gor & - & Gor & \#\# \\
\hline Amilase & $\mathrm{n} / \mathrm{a}$ & $\mathrm{n} / \mathrm{a}$ & 577,5 & 528,7 & $\mathrm{n} / \mathrm{a}$ & 647,2 & $<700^{* *}$ \\
\hline Albumina (g/L) & 34,37 & 37,62 & 36,68 & 35,7 & 48,9 & 30,3 & $26-33^{*}$ \\
\hline Proteína total (g/L) & $\mathrm{n} / \mathrm{a}$ & 70,29 & 70,09 & 66,7 & 73,86 & 73,8 & $54-71^{*}$ \\
\hline Globulinas (g/L) & $\mathrm{n} / \mathrm{a}$ & 32,67 & 33,41 & 31 & 24,96 & 43,5 & $27-44^{*}$ \\
\hline Colesterol (mg/dL) & 191 & 331,8 & 252,3 & 309,8 & 400,8 & 263,8 & $135-270^{*}$ \\
\hline Triglicerídeos (mg/dL) & 264,3 & 151,8 & 217,1 & 131,6 & $\mathrm{n} / \mathrm{a}$ & 80,1 & $33-95^{\star \star \star}$ \\
\hline ALT (U/L) & 271 & 118,4 & 77,5 & 101 & 97,4 & 291,4 & $<102^{*}$ \\
\hline AST (U/L) & 58,5 & 59,8 & $\mathrm{n} / \mathrm{a}$ & $\mathrm{n} / \mathrm{a}$ & $\mathrm{n} / \mathrm{a}$ & 107,7 & $<66^{\star \star}$ \\
\hline FA (U/L) & 53,8 & 64,5 & 57,1 & 157,9 & 109,2 & 62,2 & $<156^{*}$ \\
\hline GGT (U/L) & 33,8 & $\mathrm{n} / \mathrm{a}$ & 25,1 & 33,5 & 12,7 & 19,3 & $<6,4^{\star \star}$ \\
\hline Bilirrubina (mg/dL) & $\mathrm{n} / \mathrm{a}$ & $\mathrm{n} / \mathrm{a}$ & $\mathrm{n} / \mathrm{a}$ & $\mathrm{n} / \mathrm{a}$ & $\mathrm{n} / \mathrm{a}$ & $\mathrm{n} / \mathrm{a}$ & $0,1-0,5^{*}$ \\
\hline Uréia (mg/dL) & 34,95 & 49,51 & 42,44 & 48,4 & 94,5 & 183,5 & $21-60^{*}$ \\
\hline Creatinina (mg/dL) & $\mathrm{n} / \mathrm{a}$ & 1,0 & 1,1 & 0,99 & 1,0 & 0,7 & $0,5-1,8^{*}$ \\
\hline CPK (U/L) & 127,1 & $\mathrm{n} / \mathrm{a}$ & $\mathrm{n} / \mathrm{a}$ & $\mathrm{n} / \mathrm{a}$ & $\mathrm{n} / \mathrm{a}$ & $\mathrm{n} / \mathrm{a}$ & $<121^{\star *}$ \\
\hline Fructosamina (mmol/L) & 1,36 & 3,58 & 2,97 & 3,2 & 3,07 & 2,37 & $1,26-1,8^{\star \star *}$ \\
\hline Glicose (mg/dL) & 378 & 441 & 399 & 320 & 294 & 242 & $65-118^{*}$ \\
\hline Potássio (mEq/L) & $\mathrm{n} / \mathrm{a}$ & 5,32 & 5,53 & 5,73 & 5,18 & 5,33 & $4,0-5,0^{\star *}$ \\
\hline
\end{tabular}

(PF) polifagia, (PD) polidipisia, (PU) poliúria, (NF) normofagia, (ND) normodipsia, (NU) normúria, (s/a) sem alterações, (-) ausência, (Pi) piúria, (Ba) bacteriúria, (He) hematúria, (Pr) proteinúria, (Gor) gotículas de gordura, (n/a) não aferido. \# [34], \#\# [2], *[7], **[15], ***[29]. 


\section{Paciente 2}

Mestiço Poodle x Rottweiller, fêmea, 12 anos de idade, com diagnóstico de diabetes havia cerca de 16 meses. Sofrera ovariohisterectomia prévia e estava em tratamento com insulina humana NPH (15 UI BID) associado a três refeições diárias. A paciente apresentava sinais clínicos de adequado controle da doença. Tinha tumores de mama, otite externa crônica e placa dentária bem pronunciada (Tabela 2). A dose de Caninsulin foi de 26 UI SID. Houve sinais clínicos e labora- toriais de descontrole glicêmico após 8 dias de tratamento, aumentando-se então a dose de Caninsulin para 28 UI SID. O proprietário relatou ocorrência de fezes em maior volume e de coloração amarelada com 30 dias de tratamento associado a uma boa resposta clínica ao aumento na dose. Depois de 47 dias de tratamento com Caninsulin, a paciente desenvolveu quadro clínico de cetoacidose diabética evidenciada por respiração tipo Koussmaul, vômitos e inapetência. Foi instituída terapia emergencial com insulina regular

Tabela 2. Parâmetros clínico-laboratoriais na paciente 2 em diferentes períodos após tratamento com Caninsulin SID, e após 22 dias de retomar a terapia insulínica com insulina humana NPH BID.

\begin{tabular}{|c|c|c|c|c|c|c|}
\hline & Dia 0 & Dia 8 & Dia 30 & Dia 47 & $\begin{array}{l}22 \text { dias } \\
\text { após NPH }\end{array}$ & $\begin{array}{l}\text { Valores de } \\
\text { referência }\end{array}$ \\
\hline Peso corporal $(\mathbf{k g})$ & 22,5 & 22,0 & 23,5 & 21,5 & 24,5 & \\
\hline Sinais clínicos & NF/NU/ND & PF/PU/PD & NF/UN/ND & HF/PU/PD & NF/NU/ND & NF/NU/ND \\
\hline Hemograma & $\mathrm{s} / \mathrm{a}$ & s/a & Eosinofilia & Anemia Ncit-Ncro & s/a & \# \\
\hline \multicolumn{7}{|l|}{ Urinálise } \\
\hline Densidade & 1.033 & 1.040 & 1.035 & 1.027 & 1.021 & $1.020-1.045 \#$ \\
\hline Glicosúria (mg/dL) & 1000 & 2000 & 1000 & 1000 & 1000 & - \# \\
\hline Cetonúria (mg/dL) & - & 15 & - & - & - & $-\#$ \\
\hline Sedimento & 0-5 CP/campo & - & $\begin{array}{c}\text { 3-10 CP, } \\
\mathrm{CV} / \text { campo Pi/He }\end{array}$ & $\begin{array}{l}\text { Ba, 0-5 CP, } \\
\text { CV/campo }\end{array}$ & $\mathrm{Pi} / \mathrm{Ba} / \mathrm{He} / \mathrm{Pt}$ & \#\# \\
\hline Amilase & 947,5 & $\mathrm{n} / \mathrm{a}$ & $\mathrm{n} / \mathrm{a}$ & 1077 & 844,4 & $<700^{\star \star}$ \\
\hline Albumina (g/L) & 30,09 & 23,33 & 23,24 & 27,7 & 36,33 & $26-33^{*}$ \\
\hline Proteína total (g/L) & 80,54 & 73,57 & 62,41 & 60,4 & 70,48 & $54-71^{*}$ \\
\hline Globulinas (g/L) & 50,45 & 50,24 & 39,21 & 32,7 & 34,15 & $27-44^{*}$ \\
\hline Colesterol (mg/dL) & 469,8 & 505,8 & 473,6 & 372 & 293,5 & $135-270^{*}$ \\
\hline Triglicerídeos (mg/dL) & 140,1 & 173,5 & 74,98 & 83,6 & 124,3 & $33-95^{\star \star \star}$ \\
\hline ALT (U/L) & 189,6 & 133,6 & 169 & 81,2 & 190,7 & $<102^{*}$ \\
\hline AST (U/L) & 59,5 & $\mathrm{n} / \mathrm{a}$ & $\mathrm{n} / \mathrm{a}$ & $\mathrm{n} / \mathrm{a}$ & $\mathrm{n} / \mathrm{a}$ & $<66^{\star *}$ \\
\hline FA (U/L) & 365,6 & 299,4 & 646 & 796,6 & 399,5 & $<156^{*}$ \\
\hline GGT (U/L) & 9,5 & 8,5 & 5,1 & 8,4 & 7,6 & $<6,4^{\star *}$ \\
\hline Bilirrubina (mg/dL) & 0,5 & $\mathrm{n} / \mathrm{a}$ & $\mathrm{n} / \mathrm{a}$ & $\mathrm{n} / \mathrm{a}$ & $\mathrm{n} / \mathrm{a}$ & $0,1-0,5^{*}$ \\
\hline Uréia (mg/dL) & 27,09 & 35,33 & 13,48 & 32,4 & 34,33 & $21-60^{*}$ \\
\hline Creatinina (mg/dL) & 0,9 & 1,5 & 1,1 & 1,37 & 0,9 & $0,5-1,8^{*}$ \\
\hline CPK (U/L) & $\mathrm{n} / \mathrm{a}$ & 207,2 & $\mathrm{n} / \mathrm{a}$ & $\mathrm{n} / \mathrm{a}$ & $\mathrm{n} / \mathrm{a}$ & $<121^{* *}$ \\
\hline Fructosamina (mmol/L) & 3,18 & $\mathrm{n} / \mathrm{a}$ & 1,71 & 2,46 & 4,81 & $1,26-1,8^{\star \star *}$ \\
\hline Glicose (mg/dL) & 347 & $>600$ & 256 & 431 & 207 & $65-118^{*}$ \\
\hline Potássio (mEq/L) & 5,81 & $\mathrm{n} / \mathrm{a}$ & 5,37 & 5,92 & 5,91 & $4,0-5,0^{* *}$ \\
\hline
\end{tabular}

(PF) polifagia, (PD) polidipisia, (PU) poliúria, (NF) normofagia, (ND) normodipsia, (NU) normúria, (HF) hipofagia, (NCit/Ncro) normocítica/ normocrômica, (s/a) sem alterações, (-) ausência, (CP) células pavimentosas, (CV) células vesicais, (Pi) piúria, (Ba) bacteriúria, (He) hematúria, (Pr) proteinúria, (n/a) não aferido. \# [34], \#\# [2], *[7], **[15], ***[29]. 
cristalina, fluidoterapia, reposição de potássio e antibioticoterapia, e após resolução do quadro retornouse para terapia com insulina humana NPH na dose de 15 UI BID. Evidenciou-se pancreatite pelo aumento na atividade sérica da amilase pancreática e imagens de ultrassom. Em revisão após 22 dias do retorno à terapia insulínica com NPH humana, a paciente apresentava bom controle glicêmico. Os achados referentes a hemograma, urinálise e perfil bioquímico obtidos ao longo do período de 69 dias de acompanhamento da paciente são apresentados na Tabela 2 .

\section{Paciente 3}

Dashchund, fêmea, 8 anos de idade, com diagnóstico de diabetes seis meses antes, porém sem ter sido implementada terapia insulínica. A paciente apresentava os sinais clássicos de diabetes mellitus (Tabela 3) e alimentava-se de ração mais comida caseira. Iniciou terapia com Caninsulin na dose de 8 UI SID. Após

Tabela 3. Parâmetros clínico-laboratoriais na paciente 3 em diferentes períodos após tratamento com Caninsulin SID, e após 29 dias de iniciar terapia insulínica com insulina humana NPH BID.

\begin{tabular}{|c|c|c|c|c|c|c|}
\hline & Dia 0 & Dia 7 & Dia 15 & Dia 28 & $\begin{array}{c}29 \text { dias após } \\
\mathrm{NIH}\end{array}$ & $\begin{array}{l}\text { Valores de } \\
\text { referência }\end{array}$ \\
\hline Peso corporal (kg) & 7,0 & 7,5 & 8,3 & 7,0 & 8,3 & \\
\hline Sinais clínicos & PF/PU/PD & PF/PU/PD & NF/PU/PD & NF/PU/PD & NF/NU/ND & NF/NU/ND \\
\hline Hemograma & Leucocitose & $\mathrm{s} / \mathrm{a}$ & $\mathrm{n} / \mathrm{a}$ & $\begin{array}{c}\text { Anemia NCit/ } \\
\text { NCro, Lct c/ DER }\end{array}$ & $\begin{array}{l}\text { Anemia NCit/ } \\
\text { NCro, Lct c/ DER }\end{array}$ & $\#$ \\
\hline Urinálise & & & $\mathrm{n} / \mathrm{a}$ & & & \\
\hline Densidade & 1.060 & 1.045 & & 1.042 & 1.045 & $1.020-1.045 \#$ \\
\hline Glicosúria (mg/dL) & 1000 & 1000 & & 1000 & 1000 & $-\#$ \\
\hline Cetonúria (mg/dL) & 40 & 15 & & - & - & - \# \\
\hline Sedimento & $\begin{array}{l}\text { Pt, 0-5 } \\
\text { CP/campo }\end{array}$ & - & & - & - & \#\# \\
\hline Amilase & 2015 & $\mathrm{n} / \mathrm{a}$ & $\mathrm{n} / \mathrm{a}$ & $\mathrm{n} / \mathrm{a}$ & 1216 & $<700^{* *}$ \\
\hline Albumina (g/L) & 33,31 & $\mathrm{n} / \mathrm{a}$ & 29,46 & 20,63 & 22,8 & $26-33^{*}$ \\
\hline Proteína total (g/L) & 74,82 & $\mathrm{n} / \mathrm{a}$ & 77,86 & 66,76 & 91,54 & $54-71^{*}$ \\
\hline Globulinas ( $g / L)$ & 41,51 & $\mathrm{n} / \mathrm{a}$ & $\mathrm{n} / \mathrm{a}$ & 46,13 & 68,74 & $27-44^{*}$ \\
\hline Colesterol (mg/dL) & 492,8 & $\mathrm{n} / \mathrm{a}$ & 360,4 & 323,5 & 260,3 & $135-270^{*}$ \\
\hline Triglicerídeos (mg/dL) & 18,63 & $\mathrm{n} / \mathrm{a}$ & 58,6 & 72,58 & 84,24 & $33-95^{\star \star *}$ \\
\hline ALT (U/L) & 228 & $\mathrm{n} / \mathrm{a}$ & 165 & 12,9 & 23 & $<102^{*}$ \\
\hline AST (U/L) & $\mathrm{n} / \mathrm{a}$ & $\mathrm{n} / \mathrm{a}$ & $\mathrm{n} / \mathrm{a}$ & $\mathrm{n} / \mathrm{a}$ & $\mathrm{n} / \mathrm{a}$ & $<66^{* *}$ \\
\hline FA (U/L) & 541,8 & $\mathrm{n} / \mathrm{a}$ & 253,9 & 151,4 & 117,4 & $<156^{*}$ \\
\hline GGT (U/L) & 23,2 & $\mathrm{n} / \mathrm{a}$ & $\mathrm{n} / \mathrm{a}$ & 5,1 & 5,1 & $<6,4^{\star *}$ \\
\hline Bilirrubina (mg/dL) & $\mathrm{n} / \mathrm{a}$ & $\mathrm{n} / \mathrm{a}$ & $\mathrm{n} / \mathrm{a}$ & $\mathrm{n} / \mathrm{a}$ & $\mathrm{n} / \mathrm{a}$ & $0,1-0,5^{*}$ \\
\hline Uréia (mg/dL) & 26,77 & $\mathrm{n} / \mathrm{a}$ & 44,21 & 13,6 & 17,92 & $21-60^{*}$ \\
\hline Creatinina (mg/dL) & 1,0 & $\mathrm{n} / \mathrm{a}$ & 0,7 & 0,7 & 0,9 & $0,5-1,8^{*}$ \\
\hline CPK (U/L) & $\mathrm{n} / \mathrm{a}$ & $\mathrm{n} / \mathrm{a}$ & $\mathrm{n} / \mathrm{a}$ & $\mathrm{n} / \mathrm{a}$ & $\mathrm{n} / \mathrm{a}$ & $<121^{\star \star}$ \\
\hline Fructosamina (mmol/L) & 3,29 & $\mathrm{n} / \mathrm{a}$ & 3,31 & 2,28 & 2,4 & $1,26-1,8^{\star \star *}$ \\
\hline Glicose (mg/dL) & 382 & $\mathrm{n} / \mathrm{a}$ & 381 & 368 & 280 & $65-118^{*}$ \\
\hline Potássio (mEq/L) & 4,63 & $\mathrm{n} / \mathrm{a}$ & 5,33 & 4,36 & 5,14 & $4,0-5,0^{* *}$ \\
\hline
\end{tabular}

(PF) polifagia, (PD) polidipisia, (PU) poliúria, (NF) normofagia, (ND) normodipsia, (NU) normúria, (NCit/Ncro) normocítica/normocrômica, (Lct c/ DER) leucocitose com desvio a esquerda regenaritivo, (CP) células pavimentosas, (s/a) sem alterações, (-) ausência, (n/a) não aferido. \# [34], \#\# [2], *[7], **[15], ***[29]. 
15 dias de tratamento com Caninsulin, a paciente apresentou uveíte no olho esquerdo que foi tratada com Prednisona e Flurbiprofeno. Também apresentava um cisto perianal com conteúdo sanguinolento tratado com Enrofloxacina ( $5 \mathrm{mg} / \mathrm{kg}$ BID). Nesta ocasião foi aumentada a dose de Caninsulin para $10 \mathrm{UI}$ SID. Foi relatada cegueira pelos proprietários e apresentava alopecia bilateral. A terapia com Caninsulin foi descontinuada após 28 dias em decorrência do fraco controle glicêmico, evidenciado por desidratação, secreção ocular, poliúria e polidipsia. Iniciou-se terapia com insulina NPH humana (4 UI BID). Em avaliação posterior fora diagnosticado hipotireoidismo por medição de tiroxina total $(1 \mu \mathrm{g} / \mathrm{dL}$, valor de referência: 1,4-4,0). Somente após tratamento da hipofunção tireoidea com Levotiroxina ( $25 \mu \mathrm{g}$ BID) obtevese adequado controle glicêmico. Os achados referentes aos hemogramas, urinálises e perfis bioquímicos obtidos ao longo do período de 57 dias de acompanhamento da paciente são apresentados na Tabela 3.

\section{Paciente 4}

Fox Paulistinha, fêmea, 10 anos de idade, com diagnóstico de diabetes recente. A Tabela 4 mostra a apresentação inicial da paciente. Os sinais apresenta-

Tabela 4. Parâmetros clínico-laboratoriais na paciente 4 em diferentes períodos após tratamento com Caninsulin SID.

\begin{tabular}{|c|c|c|c|c|}
\hline & Dia 0 & Dia 18 & Dia 30 & $\begin{array}{l}\text { Valores de } \\
\text { referência }\end{array}$ \\
\hline Peso corporal (kg) & 4,0 & 4,5 & 4,0 & \\
\hline Sinais clínicos & PF/PU/PD & $\mathrm{PF} / \mathrm{NU} / \mathrm{ND}$ & PF/PU/PD & NF/NU/ND \\
\hline Hemograma & Anemia Ncit / Ncro & s/a & s/a & $\#$ \\
\hline \multicolumn{5}{|l|}{ Urinálise } \\
\hline Densidade & 1.034 & 1.025 & 1.032 & $1.020-1.045 \#$ \\
\hline Glicosúria (mg/dL) & 1000 & 1000 & 1000 & - \# \\
\hline Cetonúria (mg/dL) & 80 & - & 40 & - \# \\
\hline Sedimento & $\mathrm{He}$ & $\mathrm{He} / \mathrm{Pi}$ & 0-5 CP/campo & \#\# \\
\hline Amilase & $\mathrm{n} / \mathrm{a}$ & $\mathrm{n} / \mathrm{a}$ & 719,8 & $<700^{\star *}$ \\
\hline Albumina (g/L) & $\mathrm{n} / \mathrm{a}$ & $\mathrm{n} / \mathrm{a}$ & 33,6 & $26-33^{*}$ \\
\hline Proteína total (g/L) & 67,92 & $\mathrm{n} / \mathrm{a}$ & 64,3 & $54-71^{*}$ \\
\hline Globulinas ( $g / L)$ & $\mathrm{n} / \mathrm{a}$ & $\mathrm{n} / \mathrm{a}$ & 30,7 & $27-44^{\star}$ \\
\hline Colesterol (mg/dL) & 179,9 & 529,5 & 396,3 & $135-270^{*}$ \\
\hline Triglicerídeos (mg/dL) & 75,14 & 74,1 & 75,7 & $33-95^{\star \star *}$ \\
\hline ALT (U/L) & 97,8 & 501 & 148,5 & $<102^{*}$ \\
\hline AST (U/L) & $\mathrm{n} / \mathrm{a}$ & $\mathrm{n} / \mathrm{a}$ & $\mathrm{n} / \mathrm{a}$ & $<66^{\star \star}$ \\
\hline FA (U/L) & 132,3 & 361,4 & 350,3 & $<156^{*}$ \\
\hline GGT (U/L) & 11,5 & $n / a$ & 13,6 & $<6,4^{* *}$ \\
\hline Bilirrubina (mg/dL) & $\mathrm{n} / \mathrm{a}$ & $\mathrm{n} / \mathrm{a}$ & $\mathrm{n} / \mathrm{a}$ & $0,1-0,5^{\star}$ \\
\hline Uréia (mg/dL) & 11,59 & 38,82 & 21,2 & $21-60^{*}$ \\
\hline Creatinina (mg/dL) & $\mathrm{n} / \mathrm{a}$ & $\mathrm{n} / \mathrm{a}$ & 1,39 & $0,5-1,8^{\star}$ \\
\hline CPK (U/L) & $\mathrm{n} / \mathrm{a}$ & $\mathrm{n} / \mathrm{a}$ & $n / a$ & $<121^{* *}$ \\
\hline Fructosamina (mmol/L) & $\mathrm{n} / \mathrm{a}$ & $\mathrm{n} / \mathrm{a}$ & 3,85 & $1,26-1,8^{\star * \star}$ \\
\hline Glicose (mg/dL) & 473 & 486 & 658,7 & $65-118^{*}$ \\
\hline Potássio (mEq/L) & $\mathrm{n} / \mathrm{a}$ & $\mathrm{n} / \mathrm{a}$ & 6,11 & $4,0-5,0^{\star *}$ \\
\hline
\end{tabular}


vam evolução havia cerca de um mês e tinha histórico de obesidade e estresse crônico, manifestado por ansiedade por separação, dominância e agressividade. $\mathrm{O}$ animal comia ração comercial, comida caseira e frutas. A paciente apresentava sopro mitral, nódulos mamários e desidratação moderada. Iniciou-se tratamento com Caninsulin na dose de 5 UI SID. Após 18 dias em tratamento os proprietários relataram pequena melhora clínica, porém dificuldades em aplicar a insulina em virtude da agressividade da paciente que os mordia durante as aplicações. Após 30 dias de tratamento com Caninsulin, os proprietários, apesar de relatarem normalização do quadro clínico, confessaram alimentá-la fora dos horários pré-determinados. A Tabela 4 mostra os achados referentes aos hemograma, urinálise e perfil bioquímico obtidos ao longo do período de 30 dias de acompanhamento da paciente. As avaliações foram suspensas devido ao estresse das coletas e à não colaboração dos proprietários, iniciando-se com insulina NPH humana 2 UI BID.

\section{Paciente 5}

Cocker, fêmea, 11 anos de idade e diagnóstico recente de diabetes mellitus em decorrência de crise cetoacidótica. O proprietário relatou excesso de peso da paciente antes do início dos sintomas. A cadela apresentava diestro, evidenciado por citologia vaginal e histórico reprodutivo. Tinha muitos tumores mamários de múltiplos tipos histológicos, além de conjuntivite, gengivite e periodontite severas. Na consulta inicial a paciente encontrava-se em estado de embotamento mental e respiração tipo Koussmal. Após controle da cetoacidose diabética iniciou-se terapia com Caninsulin na dose de 15 UI/SID, que passou a 16 UI SID após duas semanas. Houve melhora clínica evidente e da glicemia pré-insulina (Tabela 5). Instituiu-se antibioticoterapia para um eczema úmido bastante extenso na região do pescoço e tratamento para ceratoconjunvite. Em revisão após 35 dias de tratamento com Caninsulin, a paciente apresentou sinais clínicos de cetoacidose diabética precedidos de sinais de fraco controle glicêmico, citologia vaginal de pró-estro e regresão das tumorações mamárias. Após controle da crise cetoacidótica foi suspensa a Caninsulin, instituída terapia com insulina NPH humana (9 UI/BID) e encaminhou-se para ovariohisterectomia. Foram observados nódulos no baço e esplenomegalia procedendo-se a biópsia aspirativa. Entretanto, após recuperação anestésica, a paciente iniciou quadro de depressão, fraqueza, taquipnéia, taquicardia e palidez de mucosas (choque vasculogênico) cerca de 5 horas depois da cirurgia. Iniciou-se transfusão sanguínea e fluidoterapia agressivas, porém o quadro evoluiu para óbito da paciente após três paradas cardio-respiratórias . O exame post-mortem revelou inúmeras metástases dos tumores de mama no baço, fígado, pulmão e outros órgãos sem evidências de hemorragia no coto uterino ou pedículos ovarianos. A Tabela 5 mostra os achados referentes aos hemogramas, urinálises e perfis bioquímicos obtidos ao longo do período de 35 dias de acompanhamento da paciente.

\section{DISCUSSÃO}

Estudos anteriores avaliaram o uso de insulina suína lenta para controle glicêmico de cães diabéticos [13]. Apenas 25\% de cães recebendo Caninsulin uma vez por dia foram capazes de eliminar os sinais clínicos e manter valores glicêmicos aceitáveis por pelo menos 22 horas. A administração de Caninsulin uma vez por dia falhou em manter valores glicêmicos aceitáveis por mais de treze horas em $62,5 \%$ dos animais [13]. Um estudo realizado em gatos diabéticos com Caninsulin concluiu que seria adequada para uso duas vezes por dia [20]. Estudos em humanos avaliando diferentes apresentações de insulinas (Glardine, NPH, ultralenta e Lispro) demonstram que Glardine apresenta grande potencial para uso a cada 24 horas e mimetiza o efeito da infusão subcutânea contínua de insulina, considerado gold standard na reposição de insulina basal [18].

No presente trabalho, os melhores resultados de controle da diabetes foram observados na paciente 1 , que tinha sido castrada e sofrido mastectomia como tratamento para os carcinomas mamários presentes, além de ter sido alimentada com dieta rica em fibras. Fatores como estresse, estro/diestro, corticoidoterapia, alimentação inadequada e doenças intercorrentes como hipotireoidismo, cistite, neoplasias, pancreatite, gengivite/periodontite, uveíte e infecções de pele influenciaram negativamente a terapia com Caninsulin na população avaliada. Qualquer desordem inflamatória, infecciosa ou neoplásica concomitante à diabetes mellitus apresenta enorme potencial para reduzir a eficácia da terapia insulínica $[4,5]$.

A ovariohisterectomia deve ser vista como parte fundamental do tratamento de uma paciente com diabetes mellitus [4,5]. A progesterona apresenta efeito antagônico diretamente nos receptores teciduais de insulina [4] além de promover síntese e secreção de GH pela glândula mamária, o que promove um efeito 
Tabela 5. Parâmetros clínico-laboratoriais na paciente 5 em diferentes períodos após tratamento com Caninsulin SID.

\begin{tabular}{|c|c|c|c|c|}
\hline & Dia 0 & Dia 15 & Dia 35 & $\begin{array}{l}\text { Valores de } \\
\text { referência }\end{array}$ \\
\hline Peso corporal (kg) & 12 & 12,7 & 12,5 & \\
\hline Sinais clínicos & PF/PU/PD & $\mathrm{PF} / \mathrm{UN} / \mathrm{ND}$ & HF/PU/PD & NF/NU/ND \\
\hline Hemograma & $\begin{array}{l}\text { Anemia NCit/ } \\
\text { Ncro, Lcp, Lfc }\end{array}$ & s/a & Anemia NCit/Ncro & $\#$ \\
\hline \multicolumn{5}{|l|}{ Urinálise } \\
\hline Densidade & 1.034 & 1.025 & 1.032 & $1.020-1.045 \#$ \\
\hline Glicosúria (mg/dL) & 1000 & 1000 & 1000 & - \# \\
\hline Cetonúria (mg/dL) & 80 & - & 40 & - \# \\
\hline Sedimento & Gor & $\mathrm{Pi} / \mathrm{Ba} / \mathrm{Gor} / \mathrm{Cr} \mathrm{FT}$ & $\begin{array}{c}\mathrm{Pi} / \mathrm{Ba} / \mathrm{Gor}, \\
4-10 \mathrm{CP} / \mathrm{campo}\end{array}$ & \#\# \\
\hline Amilase & 454,2 & $\mathrm{n} / \mathrm{a}$ & 705,6 & $<700^{\star *}$ \\
\hline Albumina (g/L) & 32,34 & 21,13 & 21 & $26-33^{*}$ \\
\hline Proteína total (g/L) & 97,89 & 61,52 & 60,4 & $54-71^{*}$ \\
\hline Globulinas (g/L) & 65,55 & 40,39 & 39,4 & $27-44^{*}$ \\
\hline Colesterol (mg/dL) & 659,4 & 340,5 & 383 & $135-270^{*}$ \\
\hline Triglicerídeos (mg/dL) & 1830 & 79,77 & 146,3 & $33-95^{\star \star \star}$ \\
\hline ALT (U/L) & 69 & 307 & 77,5 & $<102^{*}$ \\
\hline AST (U/L) & $\mathrm{n} / \mathrm{a}$ & $\mathrm{n} / \mathrm{a}$ & $\mathrm{n} / \mathrm{a}$ & $<66^{\star \star}$ \\
\hline FA (U/L) & 800,6 & 529,3 & 466,1 & $<156^{*}$ \\
\hline GGT (U/L) & 13,04 & $\mathrm{n} / \mathrm{a}$ & 13,6 & $<6,4^{\star *}$ \\
\hline Bilirrubina (mg/dL) & $\mathrm{n} / \mathrm{a}$ & $\mathrm{n} / \mathrm{a}$ & $\mathrm{n} / \mathrm{a}$ & $0,1-0,5^{\star}$ \\
\hline Uréia (mg/dL) & 49,7 & $\mathrm{n} / \mathrm{a}$ & 40 & $21-60^{*}$ \\
\hline Creatinina $(\mathrm{mg} / \mathrm{dL})$ & 1,2 & $\mathrm{n} / \mathrm{a}$ & 1,1 & $0,5-1,8^{*}$ \\
\hline CPK (U/L) & $\mathrm{n} / \mathrm{a}$ & $\mathrm{n} / \mathrm{a}$ & $\mathrm{n} / \mathrm{a}$ & $<121^{* *}$ \\
\hline Fructosamina (mmol/L) & 3,27 & 2,94 & 2,63 & $1,26-1,8^{\star \star \star}$ \\
\hline Glicose (mg/dL) & 501 & 476 & 506,8 & $65-118^{*}$ \\
\hline Potássio (mEq/L) & 6,24 & $\mathrm{n} / \mathrm{a}$ & 5,62 & $4,0-5,0^{\star \star}$ \\
\hline $\begin{array}{l}(\mathrm{PF}) \text { polifagia, }(\mathrm{PD}) \text { polidip } \\
\text { normocítica/normocrômica } \\
\text { bacteriúria, (Cr FT) cristalúr } \\
\text { não aferido. \# [34], \#\# [2 }\end{array}$ & $\begin{array}{l}\text { (PU) poliúria, } \\
\text { p) leucopenia, } \\
\text { fosfato triplo, } \\
\text { ], **[15], *** }\end{array}$ & $\begin{array}{l}\text { normodipsia, } \\
\text { c) linfocitose, } \\
\text { células pavime }\end{array}$ & $\begin{array}{l}\text { J) normúria, (HF) } \\
\text { gotículas de gor } \\
\text { as, (s/a) sem altera }\end{array}$ & $\begin{array}{l}\text { fagia, }(\mathrm{NCit} / \mathrm{Ncro} \\
,(\mathrm{Pi}) \text { piúria, }(\mathrm{Ba} \\
(-) \text { ausência, }(\mathrm{n} / \mathrm{a}\end{array}$ \\
\hline
\end{tabular}

hiperglicemiante antagônico ao efeito da insulina [30,32]. O histórico de ciclos estrais irregulares apresentado pela paciente 5 pode ter causado exaustão das células betapancreáticas, devido a estímulo progesterônico repetido [30]. Os frequientes estros nesta condição podem ter sido causa do crescimento de tumores de mama em decorrência de elevada atividade estrogênica, associada a um efeito parácrino do GH mamário produzido durante o diestro [32].
O sobrepeso apresentado por algumas pacientes antes do diagnóstico, associado à idade avançada e diestro são importantes fatores implicados com início da diabetes mellitus em cães [28]. O benefício das dietas ricas em fibras empregadas de forma conjunta à terapia insulínica para cães diabéticos tem sido demonstrada por alguns autores $[8,16,26]$.

Neoplasias intercorrentes ao tratamento do diabetes mellitus apresentam grande potencial de antago- 
nismo à insulina, especialmente se houver disseminação de metástases [4], como observado na paciente 5. Outros estudos apresentam neoplasias diversas, especialmente de mama, otites, cistites e infecções de pele como as principais afecções concomitantes à diabetes mellitus em cães $[12,28]$.

O estresse é um fator de risco importante no desenvolvimento da diabetes mellitus e da resistência à insulina [9,24,27]. A deficiência nos mecanismos glicorregulatórios durante o estresse foi demonstrada em cães diabéticos [24]. $\mathrm{O}$ uso de drogas diabetogênicas como corticóide, mesmo os de uso tópico, deve ser evitado em pacientes diabéticos [5]. Nesses casos, o incremento na atividade gliconeogênica causado por essas drogas leva a maior exigência de insulina para a manutenção da glicemia [33].

Há relatos de que deficiência de hormônios tireoidianos em pacientes diabéticos cria resistência à insulina, provavelmente por defeitos pós-receptor sobre o metabolismo da glicose [3,31]. A condição de obesidade e hiperlipidemia secundária ao hipotireoidismo representam um fator adicional de risco para o desenvolvimento da diabetes e de resistência à insulina ao longo do tratamento em pacientes diabéticos [4,21].

A crise de pancreatite apresentada pela paciente 2 foi responsável pelo quadro de perda do controle glicêmico. O estado diabético predispõe a pancreatite por hiperlipidemia associada [4]. A hipoproteinemia associada a hipercolesterolemia crônica, elevada atividade sérica da enzima amilase pancreática antes do início do tratamento com Caninsulin, e o relato do proprietário de que as fezes estavam em maior volume e com coloração amarelada de certa forma indicavam que poderia haver uma moléstia pancreática em curso [15].

$\mathrm{O}$ painel bioquímico inicial da paciente 3 sugere afecção pancreática envolvida na etiologia da diabetes mellitus. No entanto, a hipercolesterolemia associada a anemia e alguns sinais clínicos neste caso corroboraram para o diagnóstico de hipotireoidismo intercorrente [11].

Nos casos que apresentaram cistite houve prejuízo na resposta do paciente à terapia com Caninsulin. O estado diabético predispõe à formação de cistites e diversos outros quadros infecciosos em decorrência da glicosúria [23] e da redução na função dos neutrófilos [17]. O quadro de cistite representa um fator antagônico à insulina por estimular a liberação de hormônios diabetogênicos como cortisol e adrenalina, devendo receber tratamento adequado [4].
Aumentos de colesterol e fructosamina séricos, observadas ao longo dos tratamentos, estão relacionados a reduzida efetividade da terapia insulínica [25] e a hiperglicemia crônica [14], respectivamente. Altos níveis de ácidos graxos livres não esterificados (NEFA) também estão implicados na condição de resistência à insulina [22] e são observados na obesidade. Foram observadas reduções de fructosamina em algumas pacientes associadas a redução de albumina. Uma vez que a albumina responde por cerca de $60 \%$ das proteínas plasmáticas, o teor de fructosamina está influenciado pelo teor de albumina [14,15], de forma que redução na concentração de fructosamina pode não refletir melhor controle glicêmico nestes casos.

$\mathrm{O}$ estado diabético e suas conseqüências hepatobiliares refletem-se na atividade sérica das enzimas ALT, AST, FA e GGT [28] que aumentam em decorrência da lipidose hepática e a colestase correlacionadas à doença [15]. Houve grande flutuação na atividade destas enzimas nos casos estudados. Na presença de hipotireoidismo, apesar de não ter ocorrido remissão dos sinais clínicos nem manutenção da glicemia em valores adequados, a terapia insulínica com Caninsulin promoveu sensível redução na atividade das enzimas ALT, FA e GGT. A hiperlipidemia não foi reduzida satisfatoriamente em todos os casos no período estudado, o que reflete a persistência de um estado catabólico a despeito da terapia insulínica [4]. Em alguns casos houve hipercalemia decorrente do mecanismo de correção da cetoacidificação do plasma que ocorre na diabetes mellitus, através da troca de íons $\mathrm{H}^{+}$do meio extracelular por íons $\mathrm{K}^{+}$do meio intracelular $[6,15]$.

A ausência de glicose na urina antes da aplicação de insulina reflete risco de hipoglicemia [4], o que não foi observado neste estudo. A densidade específica da urina permaneceu elevada nos animais estudados refletindo glicosúria e celularidade detectadas $[4,28]$. A presença de corpos cetônicos na urina é um indicador sensível de cetoacidose [34]. Os traços de cetonúria observados ao longo da avaliação da paciente 1 podem ser em decorrência do jejum prolongado de 16 horas [15].

No caso do hemograma, as principais alterações observadas foram em decorrência de doenças intercorrentes como hipotireoidismo e infecções [4].

\section{CONCLUSÕES}

A utilização de insulina suína lenta uma vez por dia confere um razoável controle da diabetes 
Pöppl A.G., Oliveira S.T., Sortica M.S., Ferreira R.R., Barbosa P.R., Lacerda L.A.\& González F.D. 2006. Avaliação clínico-laboratorial

mellitus canina, evidenciado mediante avaliação clínico-laboratorial. Recomenda-se o uso desta preparação de insulina apenas em casos onde todos os possíveis fatores antagônicos à insulina tenham sido controlados e associado a uma dieta rica em fibras.

\section{NOTAS INFORMATIVAS}

${ }^{1}$ Caninsulin, Intervet, Holanda.

${ }^{2}$ Celm CC-530, São Paulo, Brasil.

${ }^{3}$ Multistix, Bayer, Argentina.

${ }^{4}$ Labtest Diagnóstica S.A., Lagoa Santa, MG, Brasil.

${ }^{5}$ Accu-Check Active, Roche Diagnóstica, Brasil.

\section{REFERÊNCIAS}

1 Bailhache E., Nguyen P., Krempf M., Siliart B., Magot T. \& Ouguerram K. 2003. Lipoproteins abnormalities in obese insulin-resistant dogs. Metabolism. 52: 559-564.

2 Bastos C.V. \& Bicalho A.P. 2003. Sedimentoscopia em urinas caninas com caracteristicas físico-quimicas normais: qual o seu valor? Clínica Veterinária. 45: 52-56.

3 Davison L.J., Ristic J.M.E., Herrtage M.E., Ramsey I.K. \& Catchpole B. 2003. Anti-insulin antibodies in dogs with naturally occuring diabetes mellittus. Veterinary Immunology and Immunopathology. 91: 53-60.

4 Feldman E.C. \& Nelson R.W. 2004. Canine and Feline Endocrinology and Reproduction. 3. ed. Missouri: Saunders. 1089p.

5 Fleeman L.M. \& Rand J.S. 2001. Managament of canine diabetes. Veterinary Clinics of North America: Small Animal Practice. 31: 855-879.

6 Ganong W.F. 2005. Review of Medical Physiology. 22. ed. Connecticut: Lange. 912p.

7 González F.D.H., Carvalho V., Möller V.A. \& Duarte F.R. 2001. Perfil bioquímico sangüíneo de cães e gatos na cidade de Porto Alegre, Rio Grande do Sul, Brasil. Arquivos da Faculdade de Veterinária UFRGS. 29: 1-6.

8 Graham P.A., Maskell I.E., Rawlings J.M., Nash A.S. \& Markwell P.J. 2002. Influence of a high fibre diet on glycaemic control and quality of life in dogs with diabetes mellitus. Journal of Small Animal Practice. 43: 67-73.

9 Greco D.S. 2001. Diagnosis of diabetes mellitus in dogs. Veterinary Clinics of North America: Small Animal Practice. 31: 844-853.

10 Guptill L., Glickman L. \& Glickman N. 2003. Time trends and risk factors for diabetes mellitus in dogs: analysis of veterinary medical data base records. The Veterinary Journal. 165: 240-247.

11 Hess R.S., Kass P.H. \& Van Winkle T.J. 2003. Association between diabetes mellitus, hypothyreoidism or hyperadrenocorticism, and atherosclerosis in dogs. Journal of Veterinary Internal Medicine. 17: 489-494.

12 Hess R.B., Saunders H.M., Van Winkle T.J. \& Ward C.R. 2000. Concurrent disorders in dogs with diabetes mellitus: 221 cases (1993-1998). Journal of American Veterinary Medical Association. 216: 1166-1173.

13 Horn B. \& Mitten R.W. 2000. Evaluation of an insulin zinc suspension for control of naturally occurring diabetes mellitus in dogs. Australian Veterinary Journal. 78: 831-834.

14 Jensen A.L. 1995. Glycated blood proteins in canine diabetes mellitus. Veterinary Record. 137: 401-405.

15 Kaneko J.J., Harvey J.W. \& Bruss M.C. 1997. Clinical Biochemistry of Domestic Animals. 5th edn. Missouri: Academic Press. 932p.

16 Kimmel S.E., Michel K.E., Hess R.S. \& Ward C.R. 2000. Effects of insoluble and soluble dietary fiber on glycaemic control in dogs with naturally occuring insulin-dependent diabetes mellitus. Journal of American Veterinary Medical Association. 216: 1076-1081.

17 Latimer K.S., Mahaffey E.A. 1984. Neutrophil adherence and movement in poorly and well-controlled diabetic dogs. American Journal of Veterinary Research. 45: 1498-1500.

18 Lepore M., Pampanelli S., Fanelli C., Porcellati F., Bartocci L., Vicenzo A., Cordoni C., Costa E., Brunetti P. \& Bolli G.B. 2000. Pharmacokinetic and pharmacodynamics of subcutaneous injection of long-acting human insulin analog glardine, NPH insulin, and ultralente human insulin and continuous subcutaneous infusion of insulin lispro. Diabetes. 49: 2142-2148.

19 Loste A. \& Marca M.C. 2001. Fructosamine and glycated hemoglobin in the assesment of glycaemic control in dogs. Veterinary Research. 32: 55-62.

20 Martin G.J. \& Rand J.S. 2001. Pharmacology of a $40 \mathrm{IU} / \mathrm{ml}$ porcine lente insulin preparation in diabetic cats: findings during the first week and after 5 or 9 weeks of therapy. Journal of Feline Medicine and Surgery. 3: 23-30.

21 Mattheeuws D., Rottiers M.D., Kaneko J.J. \& Vermeulen M.D. 1984. Diabetes mellitus in dogs: relationship of obesity to glucose tolerance and insulin response. American Journal of Veterinary Research. 45: 98-103.

22 McGarry J.D. 2002. Dysregulation of fatty acid metabolism in the etiology of tipe 2 diabetes. Diabetes. 51: 7-18. 
Pöppl A.G., Oliveira S.T., Sortica M.S., Ferreira R.R., Barbosa P.R., Lacerda L.A.\& González F.D. 2006. Avaliação clínico-laboratorial

23 McGuire M.C., Schulman R., Ridgway M.D. \& Bollero G. 2002. Detection of occult urinary tract infections in dogs with diabetes mellitus. Journal of the American Animal Hospital Association. 38: 541-544.

24 Miles P.D.G, Yamatani K., Lickey H.L.A. \& Vranic M. 1991. Mechanism of glucoregulatory responses to stress and their deficiency in diabetes. Procceds of National Academic Sciences. 88:1296-1300.

25 Minakata M.M., Carvalho L.S., Cardone R. \& De Marco V. 2004. Hiperlipidemia em cães com diabetes mellitus. ANCLIVEPA Brasil. 2: 48. Resumo.

26 Nelson R.W., Duesberg C.A., Ford S.L., Feldman E.C., Davenport D.J., Kiernan C. \& Neal L. 1998. Effect of dietary insoluble fiber on control of glycemia in dogs with naturally acquired diabetes mellitus. Journal of the American Veterinary Medical Association. 212: 380-386.

27 Neuvians T.P. \& Berger M. 2002. Diabetes care in cats and dogs. Diabetic Medicine. 19: 77-79.

28 Pöppl A.G. \& González F.D.H. 2005. Aspectos epidemiológicos e clínico-laboratoriais da diabetes mellitus em cães. Acta Scientiae Veterinarie. 33: 33-40.

29 Pöppl A.G., Veiga A., González F.D.H. 2005. Patologia clínica em cães com diabetes mellitus: valores de referência para triglicerídeos e fructosamina. Revista da Universidade Rural, Série Ciências da Vida. 25: 145-146.

30 Rijnberk A., Kooistra H.S. \& Mol J.A. 2003. Endocrine diseases in dogs and cats: similarities and differences with endocrine diseases in humans. Growth Hormone and IGF Reasearh. 13: S158-S164.

31 Schulman R.L. 2003. Insulin and others therapies for diabetes mellitus. Veterinary Medicine. April: 334-347.

32 Selman P.J., Mol J.A., Rutteman G.R. \& RijnberkA. 1994. Progestin treatment in the dog I. Effects on growth hormone, insulilike growth factorI anf glucose homeostasis. European Journal of Endocrinology. 131: 413-421.

33 Shaepherd P.R. \& Kahn B.B. 1999. Glucose tranporters and insulin action. The New England Journal of Medicine. 341 : 248-257.

34 Thrall M.A. 2004. Veterinary Hematology and Clinical Biochemistry. Maryland: Lippincott Williams \& Wilkins, 518 p. 35 Trapo S.M. \& Neta J.H. 2005. Complicações da insulinoterapia em cães com diabete melito. MedVep. 3: $123-127$. 